\title{
Implantable audiologic devices and facial plastic surgery in elderly patients
}

\begin{abstract}
Introduction: The goal of this paper is to describe a previously unreported etiology for audiologic implant extrusion. It is necessary to place the implant closer to the cochlea for the correct conduction of the sound. This paper represents the first reported case of audiologic device extrusion secondary to previous rhytidectomy.
\end{abstract}

Material and methods: We present a case report of a 69 -year-old patient with a history of rhytidectomy for aesthetic reasons 15 years ago. He was underwent transcutaneous active osseointegrated insertion, approximately 5 months later developed a $2 \mathrm{~cm}$ post-auricular soft tissue necrosis and partial extrusion. It was required a surgical intervention to solve the problem.

Results: Exposure of otological implants requires medical/surgical intervention with antibiotic therapy, with or without replacement of such devices as well as the realization of rotational flaps to cover the skin defects.

Conclusion: Previous periauricular surgery such as a parotidectomy or a rhytidectomy should be considered when select a patient to implant an otological device. In the case of osseointegrated implants, the percutaneous device could cause more skin problems than the transcutaneous one, which would lead us to choose one over another. In case of cochlear implants, should be located more posterior, away from the conflicting retroauricular area. Obviously as older as our patients get, higher are the chances to suffer those kind of complications. This complication has not been described previously, and should be analyzed in the preoperatory time in order to avoid bad results.
Volume 2 Issue 3 - 2017

\author{
Sarría Echegaray PL,Til Pérez G,Arancibia \\ Tagle D, Carnevale $C$ and Tomás Barberán \\ Department of Otorhinolaryngology and Head and Neck \\ Surgery, Son Espases University Hospital, Spain
}

Correspondence: Pedro Sarría Echegaray, Department of Otorhinolaryngology and Head and Neck Surgery, Son Espases University Hospital, Spain, Email pedrosarria@orlmallorca.com

Received: August 29, 2017 | Published: October 16, 2017

\section{Introduction}

Advances in technology have led to the development of several implantable bone conduction devices (BCD) employing bone conduction stimulation for the treatment and rehabilitation of mixed and conductive hearing loss. ${ }^{1} \mathrm{BCD}$ are alternatives when the benefit of conventional prosthetic equipment is limited or contraindicated. ${ }^{1,2}$ BCDs directly stimulate the inner ear by skull vibration. ${ }^{1}$ Bone conduction devices can feature either transcutaneous transmission, the externally audio processor is magnetically coupled to an implanted transducer; or percutaneous transmission, the audio processor is snapped on to a skin-penetrating implant. ${ }^{1,3}$

The complications from the implantation procedure are like those of other implantable prosthesis, including a cochlear implant These may be major complications, exceptional, requiring re-operation or hospitalization, or minor ones, which are often solved in the office ${ }^{4}$ minor adverse event are present in less than $5 \%$ of cases. ${ }^{2}$ The most common (non-device-related) complications of cochlear implant (CI) are skin flap necrosis, infection, dehiscence and device extrusion. ${ }^{5}$ The etiologies for extrusion are poor surgical technique, flap necrosis, and infection. Some cases of extrusion reported seem to be delayed in onset and associated with negative wound culture results, which challenges the established etiologies and suggests a possible alternative causality. ${ }^{6,7}$

Many patients are electively operated to solve a pathology or to correct a cosmetic defect In Head and Neck area. That is why we must always keep in mind that some of them may have more than one surgery in a very close area. In a minority of cases can compromise or alter vascularization which can lead to a poor surgical outcome. The pre and retro auricular approach for Parotidectomy and Rhytidectomy, as well as the steps of the surgery, alters vascularization of soft tissue to cover the implant.

We report one case of hearing devices extrusion secondary to previous head and neck surgery. To our knowledge, this is the first reported case of extrusion due to previous head and neck surgery.

\section{Case report}

A 69-year-old woman with a history of rhytidectomy fifteen years ago for aesthetic reasons was initially presented with congenital right-sided deafness and acquired left-sided mixed hearing loss. After appropriate work up, she underwent uncomplicated insertion of a osseointegrated transcutaneous device (BoneBridge ${ }^{\circledR}$ (BB) from MED-EL Company Innsbruck Austria). Her follow-up course was uneventful for 5 months, at this time she presented with $2 \mathrm{~cm}$ area of soft tissue necrosis over the device. It began to extrude (Figure 1). It was performed a wound debridement, without removal, soft tissue reduction and closure with a rotational flap (Figure 2). At 24 months post-operatively, she has no evidence of complications and the device is completely cover. 


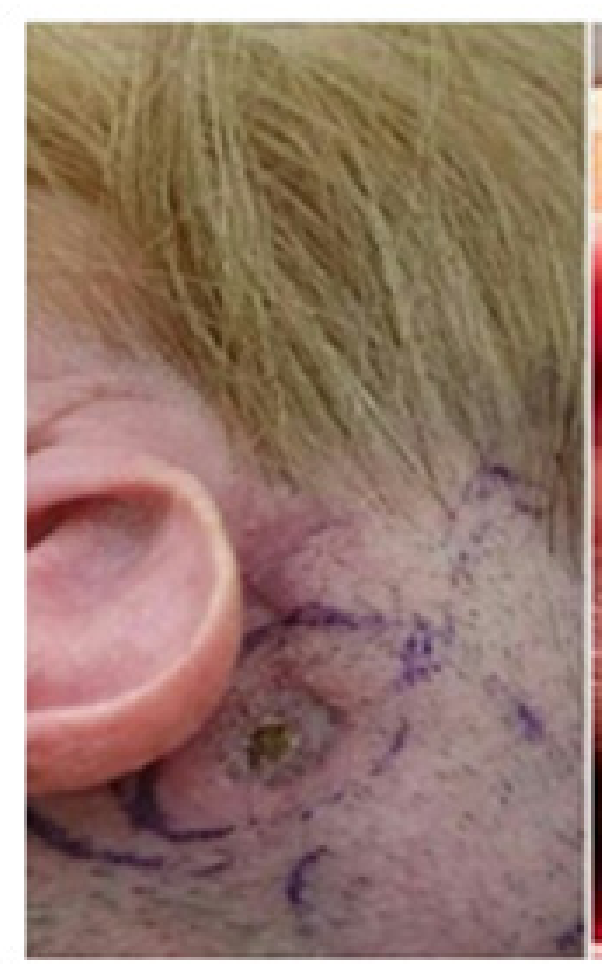

Figure I Extrude of soft tissue necrosis over the device.

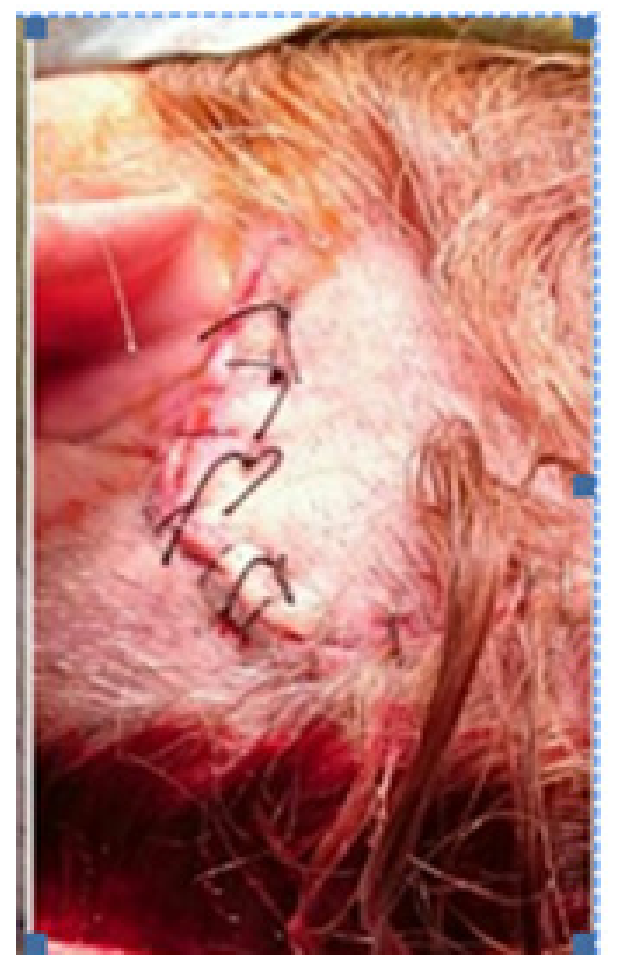

Figure 2 Soft tissue reduction and closure with a rotational flap.

\section{Discussion}

BCD implantation has been considered as a safe and reliable operation The complications that may arise from these procedures are similar to those of other implantable prosthesis, including a cochlear implant (CI). ${ }^{4}$ Wound breakdown rates of up to 10 per cent have been reported following CI insertion. ${ }^{8,9}$ BoneBridge $^{\circledR}$ (BB) is the only active and transcutaneous prosthesis, it has lowest weight and lowest external profile, this feature reduces the chances of injury to the skin. ${ }^{4}$

Skin flap necrosis is one of major complications. It can be caused by infection, hematoma, shape of the flap, or other abnormalities. The technique for skin incision is a predisposing factor of necrosis. Smaller incisions with smaller skin flaps are used to reduce vascular compromise and minimize the risk of flap necrosis. ${ }^{10,11}$ The flap thickness is very important too, to prevent breakdown and potential device extrusion, various authors recommend flaps which are 6-7 mm thick. ${ }^{12}$ Infections or post-implantation hypersensitivity to silicone could be the cause of flap necrosis. If hypersensitivity patients show no response to treatment with antibiotics, and wound cultures are negative for bacterial growth, skin patch testing to the silicone components of the implants could be performed to demonstrate sensitivity. ${ }^{13}$ In our case all the allergy tests were negative.

Bonebridge is a solution for patients with conductive/mixed hearing loss and Sensorial Sudden Deafness with different surgical approaches, depending on their anatomy. The system imparts fewer complications than percutaneous bone conduction implants and shows proven benefits in speech discrimination and functional gain. ${ }^{14}$ In our experience after $25 \mathrm{BB}$ we have only reported the complication that we present. As was seen in our patient, previous rhytidectomy is a possible cause of delayed BB extrusion. To our knowledge, this is the first reported case. It is necessary to place it closer to the cochlea for the correct conduction of the sound so we cannot avoid the conflict zone. If we treat old patients is possible to find some of them with previous facial plastic surgery or another kind of surgery than can alter the vascularization of our flap. This situation must be taken into account when proposing BCD or CI surgery to prevent future complications.

\section{Acknowledgements}

None.

\section{Conflict of interest}

The authors declare that they have no competing interest.

\section{References}

1. Zernotti ME, Di Gregorio MF, Galeazzi P, et al. Comparative outcomes of active and passive hearing devices by transcutaneous bone conduction. Acta Otolaryngol. 2016;136(6):556-558.

2. Sprinzl GM, Wolf-Magele A. The Bonebridge Bone Conduction Hearing Implant: indication criteria, surgery and a systematic review of the literature. Clin Otolaryngol. 2016;41(2):131-143.

3. Stenfelt S, Goode RL. Transmission properties of bone conducted sound: measurements in cadaver heads. $J$ Acoust Soc Am. 2005;118(4):2373-2391.

4. Zernotti ME, Sarasty AB. Active Bone Conduction Prosthesis: Bonebridge(TM). Int Arch Otorhinolaryngol. 2014;19(4):343-348.

5. Geraghty M, Fagan P, Moisidis E. Management of cochlear implant device extrusion: case series and literature review. $J$ Laryngol Otol. 2014;128(2):S55-58.

6. Harada T, Ishida K, Endo M, et al. Recurrent extrusion of cochlear implant at an interval of 5 years. Otol Neurotol. 2003;24(1):83-85. 
7. Kunda LD1, Stidham KR, Inserra MM, et al. Silicone allergy: A new cause for cochlear implant extrusion and its management. Otol Neurotol. 2006;27(8):1078-1082.

8. Kempf H, Johann K, Lenarz T. Complications in pediatric cochlear implant surgery. Eur Arch Otorhinolaryngol. 1999;256(3):128-132.

9. Hoffman RA, Cohen NL. Complications of cochlear implant surgery. Ann Otol Rhinol Laryngol. 1995; Suppl 166:420-422.

10. Davids T, Ramsden JD, Gordon KA, et al. Soft tissue complications after small incision pediatric cochlear implantation. Laryngoscope. 2009;119(5):980-983.
11. Kim YH, Cho SI. Skin Flap Necrosis by Bone Marking with Methylene Blue in Cochlear Implantation. J Audiol Otol. 2015;19(2):108-110.

12. Parkins CW, Metzinger SE, Marks HW, et al. Management of late extrusions of cochlear implants. American Journal of Otolaryngology. 1998;19(6):768-773.

13. Loochtan MJ, Yang S, Mantravadi AV, et al. Cochlear implant extrusion secondary to keloid formation. Cochlear Implants Int. 2014;15(5):276-278

14. Zernotti ME, Sarasty AB. Active Bone Conduction Prosthesis: Bonebridge(TM). Int Arch Otorhinolaryngol. 2015;19(4):343-348. 